\title{
Hercules X-1: the positive cyclotron line energy/luminosity correlation
}

\author{
D. Vasco, D. Klochkov, and R. Staubert
}

\author{
Institut für Astronomie und Astrophysik, Sand 1, 72076 Tübingen, Germany \\ e-mail: vasco@astro.uni-tuebingen.de
}

Received 10 March 2011 / Accepted 23 June 2011

\begin{abstract}
Studies of some bright, super-Eddington transient pulsars show a negative correlation between the energy of the cyclotron resonance scattering feature (CRSF) and the bolometric luminosity. For Her X-1, using repeated RXTE observations during 1996-2005, the inverse dependence was found: the energy of the cyclotron line increases as the luminosity increases. The X-ray flux measured by the RXTE/ASM (2-10 keV) has been assumed to represent the luminosity - more precisely: the maximum X-ray flux reached during the respective $35 \mathrm{~d}$ Main-On. Here, we question whether the ASM flux is really an accurate measure of the bolometric luminosity of the source. We redetermined the energy of the cyclotron line and performed spectral fits using the combined data from the PCA $(3.5-60 \mathrm{keV})$ and HEXTE $(20-75 \mathrm{keV})$ instruments on RXTE of the same $35 \mathrm{~d}$ cycles as used in the original work to determine the bolometric flux from those spectra. We confirm the result of the original analysis that the cyclotron line energy changes by $\sim 7 \%$ for a change in flux by a factor of two.
\end{abstract}

Key words. pulsars: individual: Her X-1 - stars: neutron - binaries: general

\section{Introduction}

Her X-1 is one of the most observed and well-studied accreting binary X-ray pulsars. Since the first observation made by Uhuru, the $35 \mathrm{~d}$ periodicity of the source is well known. The $\mathrm{X}$-ray light curve shows two on-states (high X-ray flux) and two off-states (low X-ray flux), with a Main-On ( 7 orbital periods) and a Short-On ( $\sim 5$ orbital cycles), separated by two off-states ( $\sim 4 \div 5$ orbital cycles each). The maximum X-ray flux of the Main-on is higher than the maximum X-ray flux of the Short-On by a factor of three to four. The modulation of the X-ray flux is believed to be caused by the periodic obscuration of the X-ray source by the precessing disk, which is believed to be inclined and warped (Gerend \& Boynton 1976). The onset of the flux, the turn-on (often identified with $35 \mathrm{~d}$ phase 0.0 ), is believed to occur when the outer rim of the disk opens up the view to the X-ray emitting regions near the polar caps on the surface of the neutron star, while the flux decrease towards the end of the on-states is associated with the inner parts of the disk covering these regions from the observer.

Her X-1 is also the first X-ray pulsar for which a cyclotron line was discovered (Trümper et al. 1978). The discovery of this line-like feature played a key-role in the measurement of the magnetic fields, providing the first direct measurement of the $B$-field of a neutron star. The energy of the cyclotron line is related to the magnetic field by the formula $B_{12}=(1+$ $z) E_{\text {cyc }} / 11.6 \mathrm{keV}$, where $B_{12}$ is the magnetic field strength in units of $10^{12}$ Gauss, $z$ is the gravitational redshift and $E_{\text {cyc }}$ is the energy of the cyclotron line. This feature is now referred to as a cyclotron resonant scattering feature (CRSF) and seems to be quite common in accreting X-ray pulsars (Coburn et al. 2002). The cyclotron line is an absorption feature produced by the resonant scattering of photons on electrons. In the $\sim 10^{12}$ Gauss magnetic field, the electrons are in quantized energy states (with respect to their movement perpendicular to the magnetic field), the socalled Landau levels. The energy levels are nearly equidistantly spaced and photons with energies equal to $n$ times the fundamental Landau energy could take part in this scattering.

In a few transient pulsars such as V0332+53 and $4 \mathrm{U} 0115+63$, a negative correlation between the CRSF and the bolometric luminosity of the source has been observed: the energy of the cyclotron line decreases as the X-ray luminosity increases (Mihara et al. 1998; Mowlavi et al. 2006; Nakajima et al. 2006; Tsygankov et al. 2006). Her X-1, however, shows the opposite behavior. Repeated measurements of the cyclotron line energy with different instruments such as RXTE and INTEGRAL in the X-ray spectrum of Her X-1 have revealed a positive correlation between the (pulse phase-averaged) cyclotron line energy $E_{\mathrm{cyc}}$ and the X-ray flux (Staubert et al. 2007), more precisely with the maximum $X$-ray flux observed during the Main-On of the respective $35 \mathrm{~d}$ cycle, as measured by RXTE/ASM in the $2-10 \mathrm{keV}$ range, which is assumed to be characteristic of the current accretion state and luminosity of the source. It has, however, been questioned whether the $2-10 \mathrm{keV}$ flux can really be taken as representative of the bolometric luminosity.

In this paper, we attempt to answer this question by reanalysing the RXTE observations of the same $35 \mathrm{~d}$ cycles as used in the original analysis of Staubert et al. (2007). Using data from both instruments (PCA and HEXTE), we re-determine the cyclotron line energy and measure the $3.5-60 \mathrm{keV}$ bolometric X-ray flux by performing spectral analyses of eleven Main-Ons between 1996 and 2005 . We show that the $2-10 \mathrm{keV}$ flux is a good measure of the bolometric flux and that the positive correlation between the phase-averaged cyclotron line energy $E_{\mathrm{cyc}}$ and the maximum $35 d$ flux, or the luminosity, is confirmed. 
Table 1. Details of RXTE observations of Her X-1 used for the spectral analysis.

\begin{tabular}{lcc}
\hline \hline $\begin{array}{l}\text { Observation } \\
\text { month/year }\end{array}$ & $\begin{array}{c}\text { 35 d Main-On } \\
\text { cycle number }\end{array}$ & $\begin{array}{c}\text { Center } \\
\text { MJD }\end{array}$ \\
\hline $\begin{array}{l}\text { July 96 } \\
\text { September 97 }\end{array}$ & 257 & 50290.75 \\
December 00 & 303 & 50707.06 \\
January 01 & 304 & 51897.69 \\
May 01 & 307 & 52933.67 \\
June 01 & 308 & 52071.16 \\
August 02 & 320 & 52492.96 \\
November 02 & 323 & 52599.32 \\
December 02 & 324 & 52634.01 \\
October 04 & 343 & 53300.95 \\
July 05 & 351 & 53577.35 \\
\hline
\end{tabular}

Notes. ${ }^{(1)}$ Cycle numbering according to Staubert et al. (2009).

\section{Observations}

Her X-1 has been repeatedly observed by RXTE since 1996 . We analyzed observations of eleven $35 \mathrm{~d}$ Main-Ons for which there were photon statistics of sufficient high quality to allow a spectral analysis. Those cycles were those of numbers (Nos.) 257, $269,303,304,307,308,320,323,324,343$ and 351. For the definition of cycle counting we refer to Staubert et al. (2009) (cycle no. 313 was not included, despite the high quality statistics, because no ASM observations were available). We cover a period of ten years of observation from July 1996 (no. 257) to July 2005 (no. 351), see Table 1. Here we used data from both RXTE instruments: PCA in the energy range $3.5-60 \mathrm{keV}$, and HEXTE in the energy range $20-75 \mathrm{keV}$.

\section{Spectral analysis}

For the spectral analysis, we used XSPEC ${ }^{1}$ (12.6.0) and generated spectra for all observed Main-Ons, summing all available data. For each spectrum (one for each Main-On), two quantities were determined: the central energy $E_{\text {cyc }}$ of the cyclotron absorption feature and the integrated flux in the range $3.5-60 \mathrm{keV}$ (in units of $\mathrm{erg} \mathrm{cm}^{-2} \mathrm{~s}^{-1}$ ). As the spectral function, we used the highecut ${ }^{1}$ model (based on a power-law continuum with an exponential cut-off) and a multiplicative Gaussian absorption line for the CRSF, in the same way as e.g. Coburn et al. (2002) and Staubert et al. (2007). Data from both RXTE instruments were used: PCA (PCU2 only) in the energy range $3.5-60 \mathrm{keV}$, and HEXTE in the energy range $20-75 \mathrm{keV}$. Cold material absorption was taken into account in each fit. All values were consistent to within two standard deviations with the mean value of $N_{\mathrm{H}}=1.08 \times 10^{22} \mathrm{~cm}^{-2}$. We note that in the original analysis PCA data were used only up to $25 \mathrm{keV}$ to define the continuum at the lower energies. New response matrices are now available $^{2}$ that allow us to use the PCA up to $\sim 50 \mathrm{keV}$, but we found that the PCA can indeed be used up to $60 \mathrm{keV}$, thereby contributing information about the cyclotron line around $40 \mathrm{keV}$. This choice is confirmed by the agreement between the PCA and HEXTE spectra in the overlapping region of the two instruments $(40-60 \mathrm{keV})$. This is also confirmed in the analysis

\footnotetext{
1 http://heasarc.gsfc .nasa.gov/docs/xanadu/xspec

2 http://www . universe.nasa.gov/xrays/programs/rxte/ $\mathrm{pca} / \mathrm{doc} / \mathrm{rmf} / \mathrm{pcarmf}-11.7$
}

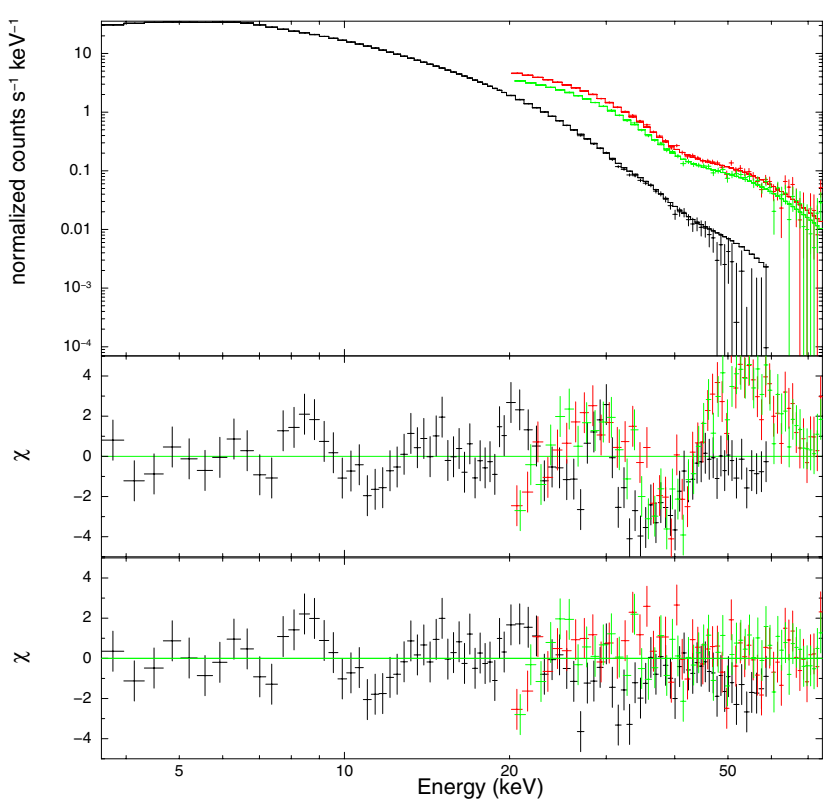

Fig. 1. Example of a spectral fit to an RXTE observation of Her X1 in July 1996 (cycle No. 257). The observation is centered at MJD 50290.75 and the integration time is $31 \mathrm{ks}$ for PCA and $10 \mathrm{ks}$ for each of the two HEXTE clusters. Black: PCA, red: HEXTE-A, and green: HEXTE-B, respectively; top: count rate spectra; middle: residuals with respect to a fit of a continuum model; bottom: residuals with respect to a fit which includes a cyclotron line.

by Rothschild et al. (2011) of RXTE observations of Cen A in which PCA data were successfully used up to $60 \mathrm{keV}$. In the new spectral fits, we also added systematic uncertainties of $0.5 \%$ (which are recommended for use with the new response matrices ${ }^{2}$ ), while in the original analysis by Staubert et al. (2007) $1 \%$ uncertainty was added.

For this spectral re-analysis, only data of RXTE PCU2 were used to ensure maximum uniformity in the data set, analyzed in a uniform way. Figure 1 shows an example of a spectral fit to data of July 1996 (cycle No. 257). The observation is centered at MJD 50290.75 and the total integration time is $31 \mathrm{ks}$ for PCA/PCU2 and $10 \mathrm{ks}$ for each of the two HEXTE clusters.

The bolometric flux for the individual spectra was found by integrating the fit function (over the $3.5-60 \mathrm{keV}$ range), using the XSPEC routine flux ${ }^{1}$. The X-ray flux of Her X-1 varies as a function of phase of the $35 \mathrm{~d}$ modulation because of variable absorption by the accretion disk.

The sampling of the various Main-Ons in groups of pointed observations was rather different, and the fluxes determined from the spectra are mean values of the observations, which happened to be made at different phases of the $35 \mathrm{~d}$ modulation. These fluxes cannot directly be compared to the maximum $X$-ray flux of the respective Main-On. To find the comparable bolometric fluxes in the $3.5-60 \mathrm{keV}$ range that represent the maximum flux for the particular cycle, it was necessary to scale the fluxes found in the individual spectra to the maximum flux. The scaling factor is the ratio of the maximum flux to the mean flux of the complete light curve, that is of the same data used to generate the spectra. To find the maximum flux, all observed RXTE/PCA light curves were fitted by a function representing a template of the mean $35 \mathrm{~d}$ Main-On modulation, the shape of which was taken from an overlay of many $35 \mathrm{~d}$ light curves observed by RXTE/ASM (see e.g., Klochkov et al. 2006). For this procedure, we are only 

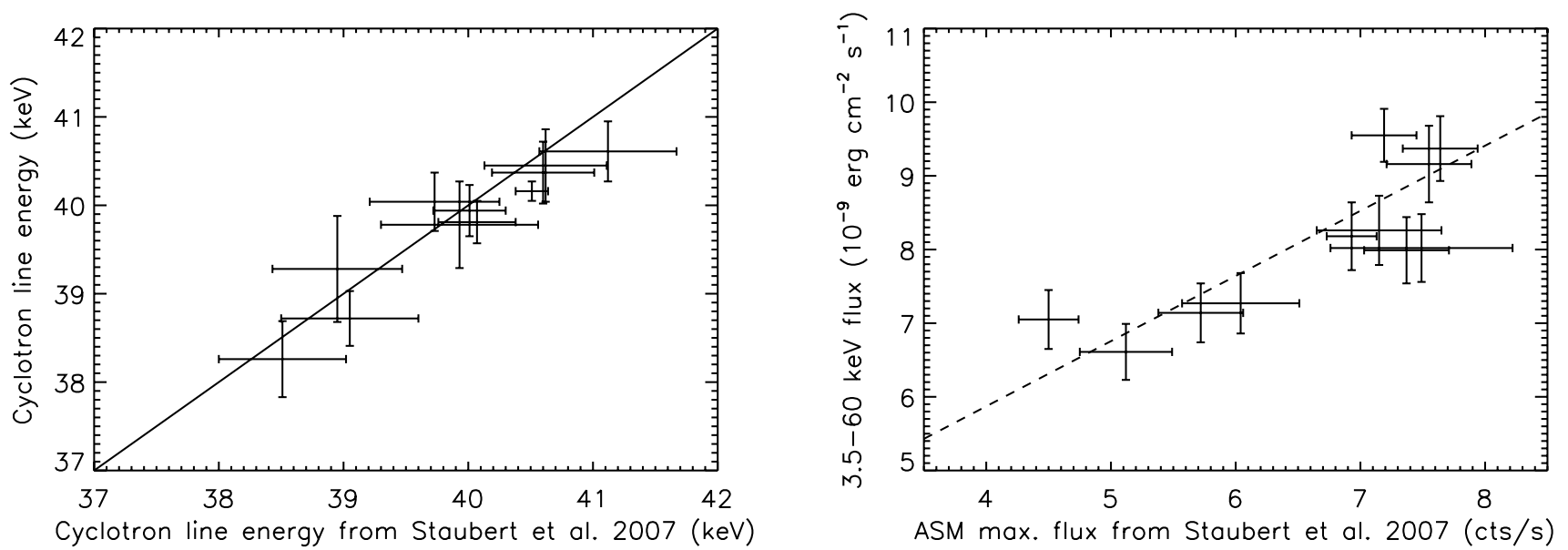

Fig. 2. Left: $E_{\text {cyc }}$ from the spectral re-analysis versus $E_{\text {cyc }}$ from the original analysis (Staubert et al. 2007). Right: scaled maximum 3.5-60 keV flux (in units of $10^{-9} \mathrm{erg} \mathrm{cm}^{-2} \mathrm{~s}^{-1}$ ) from the spectral re-analysis versus the maximum 2-10 keV ASM flux (in units of cts $\mathrm{s}^{-1}$ ) from the original analysis. The Pearson correlation coefficients are 0.95 (left) and 0.82 (right).

Table 2. Phase-averaged cyclotron line energies $E_{\mathrm{cyc}}$ and maximum fluxes reproduced from Staubert et al. (2007) (1) and from the spectral re-analysis of this work (2).

\begin{tabular}{lcccc}
\hline \hline $\begin{array}{l}35 \mathrm{~d} \mathrm{cycle} \\
\text { number }^{1}\end{array}$ & $\begin{array}{c}E_{\text {cyc }}(1)[\mathrm{keV}] \\
\text { (Staubert et al. 2007) }\end{array}$ & $\begin{array}{c}\text { max. flux }(2-10 \mathrm{keV}) \\
\left.\text { ASM [cts s}{ }^{-1}\right] \\
\text { (Staubert et al. 2007) }\end{array}$ & $\begin{array}{c}E_{\text {cyc }}(2)[\mathrm{keV}] \\
\text { (this work) }\end{array}$ & $\begin{array}{c}\text { max. flux }(3.5-60 \mathrm{keV})^{2} \\
{\left[10^{-9} \mathrm{erg} \mathrm{cm}^{-2} \mathrm{~s}^{-1}\right]} \\
\text { (this work) }\end{array}$ \\
\hline 257 & $41.12 \pm 0.55$ & $7.37 \pm 0.34$ & $40.61 \pm 0.34$ & $7.99 \pm 0.45$ \\
269 & $40.62 \pm 0.49$ & $7.49 \pm 0.73$ & $40.45 \pm 0.41$ & $8.02 \pm 0.46$ \\
303 & $40.07 \pm 0.31$ & $6.04 \pm 0.47$ & $39.81 \pm 0.24$ & $7.27 \pm 0.41$ \\
304 & $39.05 \pm 0.55$ & $5.72 \pm 0.34$ & $38.72 \pm 0.31$ & $7.14 \pm 0.40$ \\
307 & $39.93 \pm 0.63$ & $7.15 \pm 0.50$ & $39.78 \pm 0.49$ & $8.26 \pm 0.47$ \\
308 & $39.73 \pm 0.52$ & $6.93 \pm 0.20$ & $40.04 \pm 0.33$ & $8.18 \pm 0.46$ \\
320 & $40.01 \pm 0.29$ & $7.19 \pm 0.26$ & $39.94 \pm 0.29$ & $9.55 \pm 0.36$ \\
323 & $40.51 \pm 0.13$ & $7.64 \pm 0.30$ & $40.16 \pm 0.11$ & $9.37 \pm 0.44$ \\
324 & $40.60 \pm 0.41$ & $7.55 \pm 0.34$ & $40.37 \pm 0.35$ & $9.16 \pm 0.52$ \\
343 & $38.51 \pm 0.51$ & $4.50 \pm 0.24$ & $38.26 \pm 0.43$ & $7.05 \pm 0.40$ \\
351 & $38.95 \pm 0.52$ & $5.12 \pm 0.37$ & $39.28 \pm 0.60$ & $6.61 \pm 0.38$ \\
\hline
\end{tabular}

Notes. ${ }^{(1)}$ Note that for $35 \mathrm{~d}$ cycle numbers 303 and larger the corresponding numbers in Staubert et al. (2007) are larger by 1. This reflects the observation that there must have been an extra cycle during the long anomalous low before cycle 303 . However, using the numbers as given here allows to use them in an ephemeris for a rough prediction of the $35 \mathrm{~d}$ turn-ons using a mean period of $35.88 \mathrm{~d}$. We do not doubt the physical reality of the extra cycle found by Staubert et al. (2007). ${ }^{(2)}$ The flux in Col. 5 represents the $35 \mathrm{~d}$ maximum flux in the 3.5-60 keV range (see text).

interested in the overall $35 \mathrm{~d}$ modulation, that is without eclipses and dips, which was described by the analytical function:

$f(t)=A_{0}\left(1-\frac{A_{1}}{1+\exp \left(B_{1}\left(t-C_{1}\right)\right)}\right) \frac{1}{1+\exp \left(B_{2}\left(t-C_{2}\right)\right)}$,

under the condition that negative functional values are set to zero. Here $f(t)$ is the flux as a function of time $t$ in MJD, $A_{0}$ is the amplitude in units of $(3-20 \mathrm{keV}) \mathrm{PCA} \mathrm{cts} \mathrm{s}^{-1}$ per PCU, $A_{1}=1.25, B_{1}=1.87$, and $B_{2}=0.80$ are fixed functional parameters, $C_{1}$ is the time reference in $\mathrm{MJD}$, and $C_{2}=C_{1}+8.4 \mathrm{~d}$ (this offset represents the length of the standard Main-On). The time of reference $C_{1}$ was fixed to the MJD of the turn-on that had been determined from the corresponding ASM light curve. The amplitude $A_{0}$ is the only free-fitting parameter. The maximum of the fitting function (on average found to be equal to $A_{0}-5.4 \mathrm{cts} \mathrm{s}^{-1}$ ) is then taken as the maximum flux (in units of PCA cts s ${ }^{-1}$ ) for this cycle. Dividing this maximum flux by the corresponding mean flux of the complete light curve (containing all the photons that are also used in generating the corresponding spectrum) leads to a scaling factor. Multiplying the $3.5-60 \mathrm{keV}$ flux found from the spectra by this scaling factor then leads to the maximum bolometric flux (in units of erg $\mathrm{cm}^{-2} \mathrm{~s}^{-1}$ ). These bolometric flux values can now be compared to the maximum ASM flux values used in the earlier work by Staubert et al. (2007).

To test whether the above procedure in determining the maximum bolometric fluxes could be influenced by systematic changes of the spectral shape over the $35 \mathrm{~d}$ phase, we have investigated spectra of cycle No. 323 which provides a good coverage of a complete Main-On. Systematic changes were only found in one spectral parameter, the power-law index, with a magnitude of $\pm 3 \%$, which corresponds to a negligible relative change of the integrated flux of less than $5 \times 10^{-2} \%$.

The uncertainties in the bolometric flux values are dominated by the uncertainties in the scaling factors, which in turn are dominated by systematic uncertainties in determining the maximum Main-On flux in fitting the observed light curve by the common analytical function. These systematic uncertainties are estimated to be $3 \%$, the main contributions being the uncertainty in the turn-on time and the fluctuations in the shape of the modulation from cycle to cycle. 

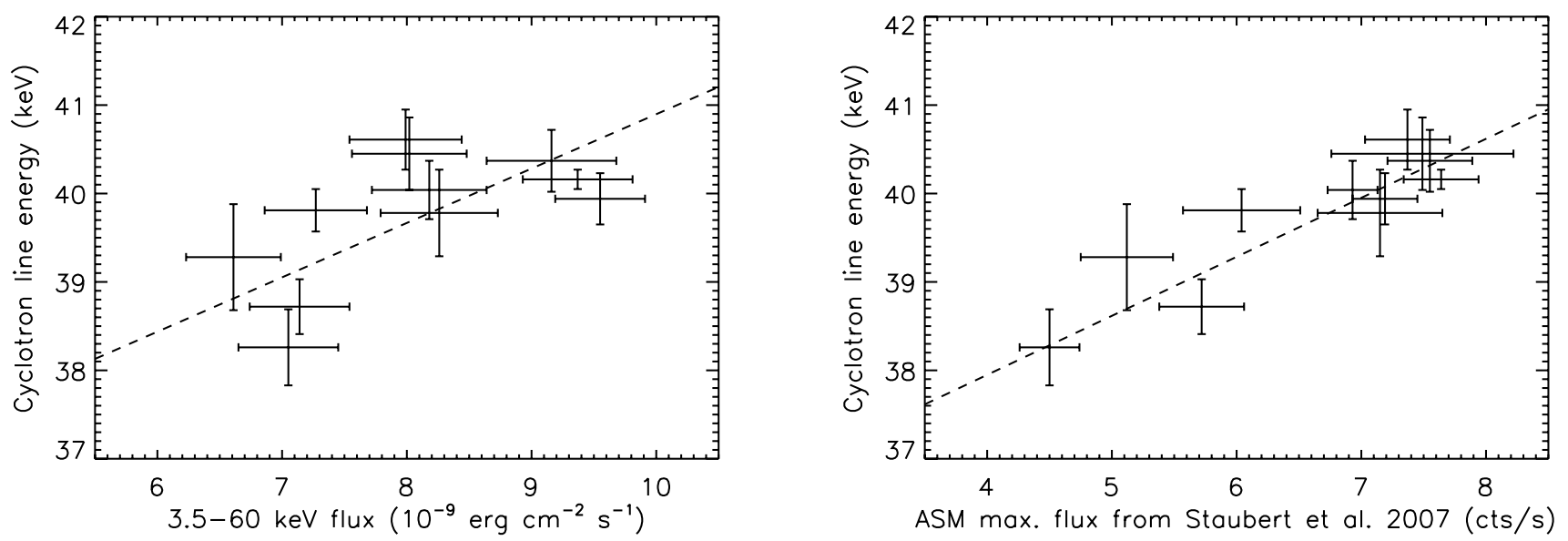

Fig. 3. Left: $E_{\mathrm{cyc}}$ from the spectral re-analysis versus the scaled maximum $3.5-60 \mathrm{keV}$ flux (in units of $10^{-9} \mathrm{erg} \mathrm{cm}^{-2} \mathrm{~s}^{-1}$ ). Right: $E_{\mathrm{cyc}}$ from the spectral re-analysis versus the maximum 2-10 $\mathrm{keV}$ ASM flux (in units of $\mathrm{cts} \mathrm{s}^{-1}$ ) from the original analysis.

Table 2 compares the results of this re-analysis to those of the original analysis by Staubert et al. (2007).

\section{Results and discussion}

In Fig. 2 (left), we plot the newly determined cyclotron line energies against those from the original analysis. Overall, we find a very good agreement between the new values for $E_{\text {cyc }}$ and those from the original analysis: considering the respective uncertainties, the differences are smaller than 0.79 standard deviations for ten of the eleven values and 2.1 standard deviations for one value (cycle No. 323).

In Fig. 2 (right), the $3.5-60 \mathrm{keV}$ maximum bolometric fluxes are plotted against the corresponding ASM fluxes of the original analysis. There is a good linear relationship between the two fluxes: the slope (taking the uncertainties in both variables into account $)$ is $0.88 \pm 0.15\left(10^{-9} \mathrm{erg} \mathrm{cm}^{-2} \mathrm{~s}^{-1}\right) /\left(\mathrm{ASM} \mathrm{cts} \mathrm{s}{ }^{-1}\right)$, and the Pearson correlation coefficient is 0.82 , corresponding to a chance probability of no correlation of $P<10^{-3}$. This demonstrates that the maximum ASM flux can be taken as a good measure of the maximum bolometric $X$-ray flux (and luminosity) of Her X-1 during the respective $35 \mathrm{~d}$ cycle. The variation in flux from one $35 \mathrm{~d}$ cycle to the next is small, such that the maximum observed flux of a particular $35 \mathrm{~d}$ Main-On can be considered a good measure of the luminosity of the source during this particular cycle. This is why the maximum ASM flux was used as a reference in the original analysis by Staubert et al. (2007).

The final correlation between the cyclotron line energy and the X-ray flux is given in Fig. 3 in two ways: we correlate $E_{\text {cyc }}$ from the re-analysis with the scaled 3.5-60 keV maximum Main-On flux (Fig. 3, left), as well as the maximum ASM flux (Fig. 3, right). The corresponding slopes of the linear fits to these data (taking the uncertainties of both variables into account) and the corresponding Pearson correlation coefficients $r$ are: (i) for the Main-On flux presented in Fig. 3 (left) slope $=0.62 \pm$ $0.19(\mathrm{keV}) /\left(10^{-9} \mathrm{erg} \mathrm{cm}^{-2} \mathrm{~s}^{-1}\right)$ and $r=0.62\left(P=2 \times 10^{-2}\right)$; (ii) for the maximum ASM flux presented in Fig. 3 (right) slope = $0.67 \pm 0.14(\mathrm{keV}) /\left(\mathrm{ASM} \mathrm{cts} \mathrm{s}^{-1}\right)$ and $r=0.90\left(P=10^{-4}\right)$.

The correlation seen in Fig. 3 (left) is somewhat less convincing than that of Fig. 3 (right) (and that in the original analysis of Staubert et al. 2007). We attribute this to the unavoidably larger uncertainties associated mainly with the scaling of the bolometric flux measured for the individual spectrum to the flux that does describe the maximum flux of the particular $35 \mathrm{~d}$ cycle. The originally used maximum ASM fluxes are, in contrast, simple direct measurements.

\section{Summary}

We have re-analyzed observations of Her X-1 in its Main-On state by RXTE between 1996 and 2005 with respect to its $\mathrm{X}$-ray spectrum. Using data from both instruments (PCA and HEXTE) we performed a spectral analysis of observations of eleven Main-Ons and determined the cyclotron line energy $E_{\text {cyc }}$ and the 3.5-60 keV flux for each of those Main-Ons. This observed flux was then scaled to a flux representing the maximum flux of the particular Main-On. We conclude that the maximum ASM flux used in the original analysis (Staubert et al. 2007) can really be taken as a measure of the luminosity of the source because it scales well with the maximum 5-60 keV flux estimated through the spectral analysis. This is evident from Fig. 2 (right) that shows there is a good linear relationship between the bolometric $3.5-60 \mathrm{keV}$ flux with the $2-10 \mathrm{keV}$ flux measured by RXTE/ASM (both fluxes refer to the maximum $35 d$ Main-On flux).

The information contained in Figs. 2 and 3 (left and right) provides an internally consistent picture. We consider the combined evidence of all the correlations shown as proof of the correctness of the positive correlation between the cyclotron line energy and source luminosity as suggested in Staubert et al. (2007). A direct comparison to the original analysis can be made by considering Fig. 3 (right): the slope of the linear best fit is determined to $(0.67 \pm 0.14) \mathrm{keV} /\left(\mathrm{cts} \mathrm{s}^{-1}\right)$, which is in good agreement with the $(0.66 \pm 0.10) \mathrm{keV} /\left(\mathrm{cts} \mathrm{s}^{-1}\right)$ found in the original analysis. The final and confirmed result with respect to this correlation can then be stated as follows: the value of the cyclotron line energy $E_{\mathrm{cyc}}$ increases by $\sim 7 \%$ for a change in flux of a factor of two.

In conclusion, we briefly describe the physical significance of the observed dependence - of either sign - of the cyclotron line energy with source luminosity. The negative correlation (decrease of $E_{\text {cyc }}$ with luminosity), which has been repeatedly observed for high luminosity transients such as V $0332+53$ and 4U 0115+63 (Mihara et al. 1998; Mowlavi et al. 2006; Nakajima et al. 2006; Tsygankov et al. 2006), has been interpreted in the 
following way: when the mass accretion rate (and hence the luminosity) increases, the height of the radiative shock above the neutron star surface increases, leading to a decrease in the effective magnetic field strength in the scattering region and therefore to a decrease in $E_{\text {cyc }}$. This is in line with theoretical considerations about accretion in the (locally) super-Eddington regime (Burnard et al. 1991). In the sub-Eddington regime, however, believed to be realized in Her X-1, the deceleration of the accreted material is thought to be due to Coulomb drag and collective plasma effects (Nelson et al. 1993). In this case, under an increased accretion rate the atmosphere is compressed by the ram pressure of the infalling material and the scattering region moves closer to the neutron star surface. This is equivalent to an increase in effective field strength and an increase in $E_{\text {cyc }}$, hence to a positive correlation with luminosity (Staubert et al. 2007).

We would also like to add that the recent analysis of Klochkov et al. (2011) supports the physical correlation between the cyclotron line energy and the luminosity of three sources: 4U $0115+63$, V 0332+53, and Her X-1. In this analysis data from a short time interval were used, and spectra were generated by summing up photons belonging to individual pulses in selected ranges of pulse amplitude. In this pulse-to-pulse variability study, the variations in X-ray flux (source luminosity) occur on timescales comparable to the duration of the individual pulses (the period of rotation of the neutron star). The previously found correlations (based on flux variations on much longer timescales) between the cyclotron line energy and the $\mathrm{X}$-ray flux are reproduced: that is a negative correlation for the super-Eddington transient sources $4 \mathrm{U} 0115+63$ and V $0332+53$, and a positive correlation for the sub-Eddington source Her X-1.

Acknowledgements. D.V. and coauthors thank DLR for financial support through grant 50 OR 0702.

\section{References}

Burnard, D., Arons, J., \& Klein, R. 1991, ApJ, 367, 575

Coburn, W., Heindl, W. A., Rothschild, R. E., et al. 2002, ApJ, 580, 394

Gerend, D., \& Boynton, P. 1976, ApJ, 209, 562

Klochkov, D. K., Shakura, N. I., Postnov, K. A., et al. 2006, Astron. Lett., 32, 804

Klochkov, D., Santangelo, A., Staubert, R., \& Rothschild, R. 2011, in Proc. 8th INTEGRAL Workshop, The Restless Gamma-ray Universe, PoS(INTEGRAL2010)061

Mihara, T., Makishima, K., \& Nagase, F. 1998, Adv. Space Res., 22, 987

Mowlavi, N., Kreykenbohm, I., Shaw, S. E., et al. 2006, A\&A, 451, 187

Nakajima, M., Mihara, T., Makishima, K., \& Niko, H. 2006, ApJ, 646, 1125

Nelson, R., Salpeter, E., \& Wassermann, I. 1993, ApJ, 418, 874

Rothschild, R. E., Markowitz, A., Rivers, E., et al. 2011, ApJ, 733, 23

Staubert, R., Shakura, N. I., Postnov, K., et al. 2007, A\&A, 465, L25

Staubert, R., Klochkov, D., Postnov, K., et al. 2009, A\&A, 494, 1025

Trümper, J., Pietsch, W., Reppin, C., et al. 1978, ApJ, 219, L105

Tsygankov, S. S., Lutovinov, A. A., Churazov, E. M., \& Sunyaev, R. A. 2006, MNRAS, 371, 19 